\title{
Predictions of chiral perturbation theory for Compton scattering off protons
}

\author{
Vadim LENSKY* \\ ECT* Trento \\ E-mail: lensky@ect.it \\ Vladimir PASCALUTSA \\ Institut für Kernphysik, Johannes Gutenberg Universität, Mainz D-55099, Germany
}

\begin{abstract}
We compute the Compton scattering off the nucleons in the framework of manifestly covariant baryon chiral perturbation theory $(\mathrm{B} \chi \mathrm{PT})$. The results for observables differ substantially from the corresponding calculations in heavy-baryon chiral perturbation theory ( $\mathrm{HB} \chi \mathrm{PT})$, most appreciably in the forward kinematics. We verify that the covariant $p^{3}$ result fulfills the forwardCompton-scattering sum rules. We also explore the effect of the $\Delta(1232)$ resonance at order $p^{4} / \Delta$, with $\Delta \approx 300 \mathrm{MeV}$, the resonance excitation energy. We find that the substantial effect of the $\Delta$-excitation on the nucleon polarizabilities can naturally be accommodated in the manifestly covariant calculation.
\end{abstract}

International Workshop on Effective Field Theories: from the pion to the upsilon 2-6 February 2009

Valencia, Spain

${ }^{*}$ Speaker. 


\section{Introduction}

Compton scattering off nucleons allows to study the structure and the e.m. properties of the nucleon. At very low energies, the process depends on the static e.m. moments of nucleon, the charge and magnetic moment [1]. At larger energies (around $100 \mathrm{MeV}$ and above), the effects of the nucleon structure can be detected [2, 3, 4, 5, 6, , , , $]$. For instance, these effects show up in the values of nucleon polarizabilities - see the PDG column of Table 1.

More insights come from chiral perturbation theory $(\chi \mathrm{PT})$, an effective theory of the lowenergy strong interaction [9 10]. The leading-order $\chi \mathrm{PT}$ result for the nucleon polarizabilities is a prediction - the low-energy constants (LECs) start to contribute at the next order. The first calculation of polarizabilities in $\chi \mathrm{PT}[11$ at leading order yields the values shown in the $\mathrm{B} \chi \mathrm{PT}$ $\mathscr{O}\left(p^{3}\right)$ column of Table 1 This calculation was done in manifestly Lorentz-covariant baryon $\chi \mathrm{PT}$ $(\mathrm{B} \chi \mathrm{PT})$, to distinguish it from the heavy-baryon $\chi \mathrm{PT}(\mathrm{HB} \chi \mathrm{PT})$, which was introduced [15] in order to cure the chiral power counting problems that $\mathrm{B} \chi \mathrm{PT}$ had apparently had [16]. Incidentally, the $\mathrm{HB} \chi \mathrm{PT}$ result agrees with experiment much better, see Table1 More detailed analyses of Compton scattering in $\mathrm{HB} \chi \mathrm{PT}$ followed [12 17, 18]. However, it was shown more recently [19 20] that

\begin{tabular}{||c|c|c||c|c||c||}
\hline \hline \multirow{2}{*}{} & \multicolumn{2}{|c|}{ HB $\chi \mathrm{PT}$} & \multicolumn{2}{c||}{ B $\chi$ PT } & PDG \\
\cline { 2 - 5 } & $\mathscr{O}\left(p^{3}\right)[12]$ & $\mathscr{O}\left(\varepsilon^{3}\right) \llbracket[13]$ & $\mathscr{O}\left(p^{3}\right)[11]$ & $\mathscr{O}\left(p^{4} / \Delta\right)$ & {$[14]$} \\
\hline$\alpha^{(p)}$ & 12.2 & 20.8 & 6.8 & 10.8 & $12.0 \pm 0.6$ \\
$\beta^{(p)}$ & 1.22 & 14.7 & -1.8 & 2.9 & $1.9 \pm 0.5$ \\
\hline \hline
\end{tabular}

Table 1: The electric $(\alpha)$ and magnetic $(\beta)$ polarizabilities of the proton in units of $10^{-4} \mathrm{fm}^{3}$. The last column quotes the PDG compilation of experimental results, while the first two represent the predictions of the $\mathrm{HB} \chi \mathrm{PT}$ and $\mathrm{B} \chi \mathrm{PT}$, respectively.

$\mathrm{B} \chi \mathrm{PT}$ does not have a problem with power counting per se. It was also pointed out [21] that the difference between $\mathrm{B} \chi \mathrm{PT}$ and $\mathrm{HB} \chi \mathrm{PT}$ results can be large due to the presence of physical cuts and other non-analytic structures. Moreover, $\mathrm{HB} \chi \mathrm{PT}$ is incompatible with the sum rules [22, 23, 24]. Finally, the effect of the $\Delta$ excitation in Compton scattering cannot be accommodated in the HB framework in a natural way [25, 26] (see also the HB $\chi$ PT column of Table 11). These observations make a strong case for adopting the $\mathrm{B} \chi \mathrm{PT}$ formalism in favor of the heavy-baryon one. Here we present the results of a calculation of Compton scattering in $\mathrm{B} \chi \mathrm{PT}$ to orders $p^{3}$ and $p^{4} / \Delta[27]$.

\section{Chiral loops and Lagrangians}

Up to $\mathscr{O}\left(p^{3}\right)$, the $\chi \mathrm{PT}$ expansion for the Compton amplitude contains the Born graphs, the Wess-Zumino-Witten anomaly (see, e.g., Ref. [26]), and the loop graphs shown in Fig. 10 To calculate these loops, we consider the leading-order chiral Lagrangian for the nucleon:

$$
\mathscr{L}_{N}^{(1)}=\bar{N}\left(i \not D-M_{N}+g_{A} \phi \gamma_{5}\right) N,
$$

where $N$ denotes the isodoublet Dirac field of the nucleon, $M_{N}$ is the nucleon mass and $g_{A}$ is the axial-coupling constant, and the chiral covariant derivative is given by $D_{\mu} N=\partial_{\mu} N+i v_{\mu} N$, 
whereas the vector and axial-vector fields above are defined in terms of the pion field, $\pi^{a}(x)$, as

$$
v_{\mu} \equiv \frac{1}{2} \tau^{a} v_{\mu}^{a}(x)=\frac{1}{2 i}\left(u \partial_{\mu} u^{\dagger}+u^{\dagger} \partial_{\mu} u\right), \quad a_{\mu} \equiv \frac{1}{2} \tau^{a} a_{\mu}^{a}(x)=\frac{1}{2 i}\left(u^{\dagger} \partial_{\mu} u-u \partial_{\mu} u^{\dagger}\right),
$$

with $u=\exp \left(i \pi^{a} \tau^{a} / 2 f\right)$, and $f$ the pion decay constant. Then, we apply a redefinition of the nucleon field, $N \rightarrow \xi N$, where $\xi$ has the form:

$$
\xi=\exp \left(\frac{i g_{A} \pi^{a} \tau^{a}}{2 f} \gamma_{5}\right) .
$$

For the one-loop contributions to Compton scattering it is sufficient to expand up to the second order in the pion field. After the expansion, the redefined Lagrangian takes the following form:

$$
\mathscr{L}_{N}^{(1)}=\bar{N}\left(i \not \partial-M_{N}-i \frac{g_{A}}{f} M_{N} \tau^{a} \pi^{a} \gamma_{5}+\frac{g_{A}^{2}}{2 f^{2}} M_{N} \pi^{2}-\frac{\left(g_{A}-1\right)^{2}}{4 f^{2}} \tau^{a} \varepsilon^{a b c} \pi^{b} \not \partial \pi^{c}\right) N+O\left(\pi^{3}\right) .
$$

Finally, one gets the set of diagrams in Fig. 1 with the couplings from Eq. 2.4 instead of the usual set [11]. However, the two sets of one-loop diagrams give identical expressions for the Compton amplitude. This fact also explains why the one-loop result for polarizabilities in the linear sigma model with heavy $\sigma$-meson [28] is exactly the same as in $\mathrm{B} \chi \mathrm{PT}$ at $\mathscr{O}\left(p^{3}\right)$ [11].

The $\Delta$ excitation starts to contribute at order $p^{4} / \Delta$, where $\Delta=M_{\Delta}-M_{N} \approx 0.3 \mathrm{GeV}$. The relevant diagrams are shown in Fig. 2. The $\Delta$ Born contribution is calculated in the same way as in Ref. [26], except that we use the values of the $\gamma N \rightarrow \Delta$ couplings $\left(g_{M}=2.95\right.$ and $\left.g_{E}=-1.0\right)$ from the pion-photoproduction analyses of Refs. [31, 32], and also include the corresponding crossed graph. We use $h_{A}=2.85$, corresponding to the $\Delta \rightarrow \pi N$ decay width of $115 \mathrm{MeV}$.

The one-particle-reducible graphs in Figs. 1 1 and 2 contribute to the nucleon mass, field, charge, and magnetic moment renormalization. We adopt the on-mass-shell renormalization scheme, and use the following values of the parameters: $e^{2} / 4 \pi=1 / 137, g_{A}=1.267, f=f_{\pi}=0.0924 \mathrm{GeV}$, $m_{\pi}=0.139 \mathrm{GeV}, M_{N}=0.9383 \mathrm{GeV}, \kappa_{N}=1.79$ for the proton.

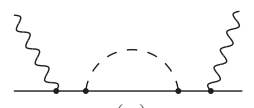

(a)
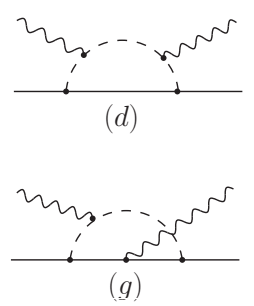

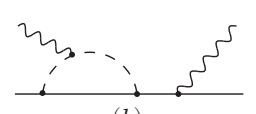

(b)

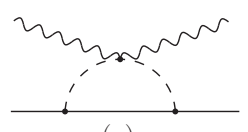

(e)

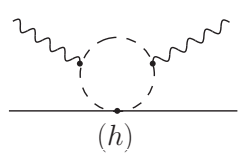

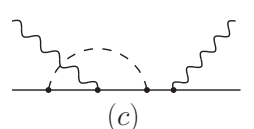

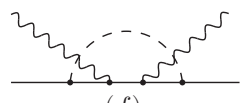

$(f)$

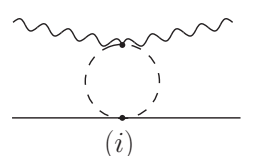

Figure 1: The loop graphs evaluated in this work. Graphs obtained from these by crossing and time-reversal are not shown, but are evaluated too.

\section{Consistency with sum rules}

The amplitude of forward Compton scattering can be related to an integral over energy of the photoabsorption cross-section, which in combination with the low-energy expansion yields a 

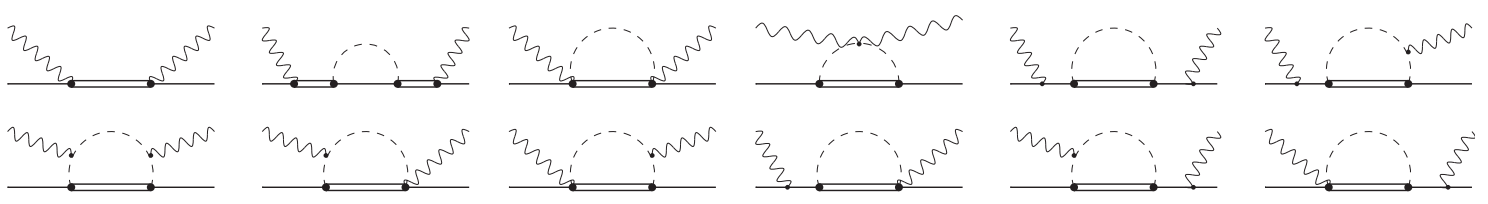

Figure 2: The graphs with $\Delta(1232)$ that contribute at order $p^{4} / \Delta$. Graphs obtained from these by crossing are not shown, but are calculated as well.

number of model-independent sum rules. A famous example is the Baldin sum rule:

$$
\alpha+\beta=\frac{1}{2 \pi^{2}} \int_{0}^{\infty} d \omega \frac{\sigma_{T}(\omega)}{\omega^{2}-i 0}
$$

that relates the sum of polarizabilities to an integral of the total photoabsorption cross-section $\sigma_{T}$.

In general, the forward Compton-scattering amplitude can be decomposed into two scalar functions of single variable:

$$
T_{f i}(\omega)=\vec{\varepsilon}^{*} \cdot \vec{\varepsilon} f(\omega)+i \vec{\sigma} \cdot\left(\vec{\varepsilon}^{*} \times \vec{\varepsilon}\right) \omega g(\omega),
$$

where $\vec{\varepsilon}^{\prime}, \vec{\varepsilon}$ are the polarization vectors of the initial and final photons, respectively, and $\vec{\sigma}$ are the Pauli spin matrices. Using analyticity and unitarity, one can write down the following sum rules:

$$
f(\omega)=f(0)+\frac{\omega^{2}}{2 \pi^{2}} \int_{0}^{\infty} d \omega^{\prime} \frac{\sigma_{T}\left(\omega^{\prime}\right)}{\omega^{\prime 2}-\omega^{2}-i 0}, \quad g(\omega)=\frac{1}{4 \pi^{2}} \int_{0}^{\infty} d \omega^{\prime} \omega^{\prime} \frac{\sigma_{1 / 2}\left(\omega^{\prime}\right)-\sigma_{3 / 2}\left(\omega^{\prime}\right)}{\omega^{\prime 2}-\omega^{2}-i 0},
$$

where $\sigma_{\lambda}$ is the doubly-polarized photoabsorption cross-section, with $\lambda$ being the helicity of the initial photon-nucleon state. We showed that the loop contributions in Fig. 1 1 fulfill the sum rules Eq. (3.3). From the sum rules, one can see that at $\mathscr{O}\left(p^{3}\right)$, chiral symmetry is not relevant for the forward Compton amplitude. The graphs $(h)$ and $(i)$ in Fig. 1 take the role of chiral symmetry. In the forward angles these graphs vanish, but play an important role in the backward angles. Without them the value of $\alpha-\beta$ would diverge as $1 / m_{\pi}^{2}$ in the chiral limit (instead of $1 / m_{\pi}$ as it should). Thus, chiral symmetry plays a more prominent role in the backward Compton scattering.

The results for the polarizabilities are in worse agreement with experiment than the $\mathrm{HB} \chi \mathrm{PT} p^{3}$ result (see Table 1). This, in fact, opens a room for the $\Delta(1232)$ contributions. The $\Delta(1232)$ plays an important role in nucleon polarizabilities, as can be seen from the Baldin sum rule and the fact that the photoabsorption cross-section is dominated, at lower energies, by the $\Delta$ resonance. In contrast, the $\operatorname{HB} \chi \mathrm{PT} p^{3}$ value for $\alpha+\beta$ saturates the sum rule, leaving no room for other contributions.

\section{Results for observables}

In Fig. 3, we show the unpolarized differential cross-section of the $\gamma p \rightarrow \gamma p$ process as a function of the scattering angle in center-of-mass system, with the incident photon energy fixed at just below the pion-production threshold. The major differences between the $\mathrm{HB} \chi \mathrm{PT}$ and $\mathrm{B} \chi \mathrm{PT}$ $p^{3}$ calculations arise at forward angles. This is because at low energies the $p^{3}$ contribution to the cross-section at forward (and backward) angles is determined by the $p^{3}$ contribution to $\alpha+\beta$ (and 
$\alpha-\beta)$. The sum of polarizabilities differs between the two calculations much more than their difference, and this fact reflects itself in the cross-section.

The red solid line with an error band in Fig. 3 shows the result of adding the $\Delta$ contribution to the covariant $p^{3}$ result. The $\Delta$ contribution in $\mathrm{B} \chi \mathrm{PT}$ is compatible with both photoproduction and Compton scattering data, which is further demonstrated in Fig. 4. where the $\gamma p \rightarrow \gamma p$ cross-section is plotted as a function of photon energies at fixed angles (in the lab system). The $\mathrm{HB} \chi \mathrm{PT}$ result is omitted here, but can be found in Ref. [18]. The results for the proton polarizabilities, complete up to $\mathscr{O}\left(p^{4} / \Delta\right)$, are displayed in Table 1

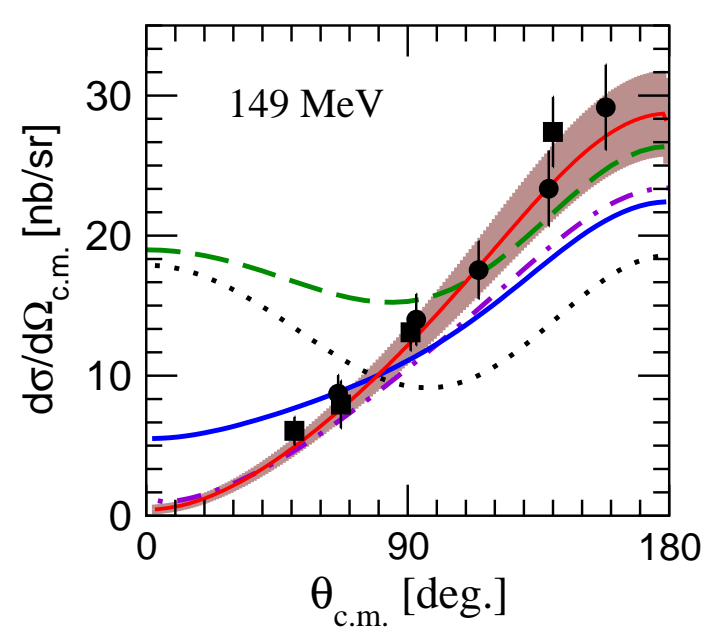

Figure 3: (Color online) Angular dependence of the $\gamma p \rightarrow \gamma p$ differential cross-section in the center-of-mass system for fixed photon-beam energy, $E_{\gamma}^{(l a b)}=149 \mathrm{MeV}$. Data points are from SAL 4] - filled squares, and MAMI [6] - filled circles. The curves are: Klein-Nishina - dotted, Born graphs and WZW-anomaly — green dashed, adding the $\mathrm{HB} \chi \mathrm{PT}$ - violet dash-dotted, adding the $\mathrm{B} \chi \mathrm{PT}-$ blue solid. The result of adding the $\Delta$-excitation contribution to the $\mathrm{B} \chi \mathrm{PT} p^{3}$ is shown by the red solid line with a band.
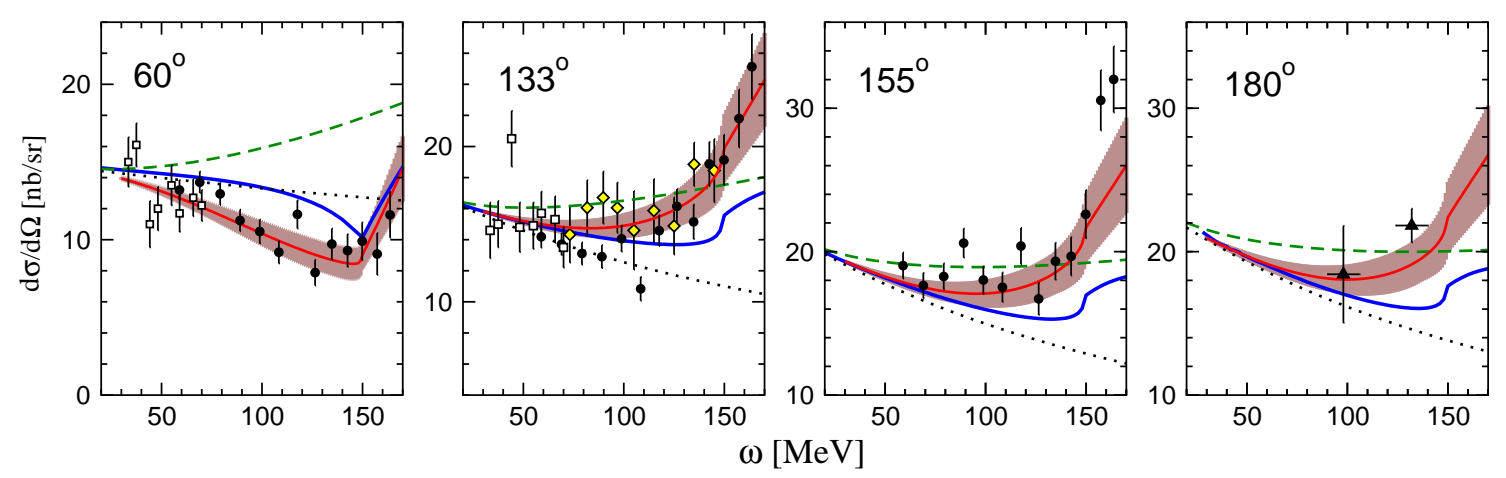

Figure 4: (Color online) Energy dependence of the $\gamma p \rightarrow \gamma p$ differential cross-section in the laboratory frame for fixed values of the scattering angle. Data: Illinois [2] - open squares, MAMI [3] - filled triangles, SAL [5] - open diamonds, and MAMI [6] - filled circles. The curves are as in Fig. 3.

\section{Conclusion}

We have studied the nucleon Compton scattering in the framework of $\mathrm{B} \chi \mathrm{PT}$ at orders $p^{3}$ and 
$p^{4} / \Delta$. The covariant $p^{3}$ result fulfills the forward-Compton-scattering sum rules. Chiral symmetry has no effect on the forward scattering but plays an important role at the backward scattering. For the $\gamma p \rightarrow \gamma p$ cross sections we find that the difference between the $\mathrm{HB} \chi \mathrm{PT}$ and $\mathrm{B} \chi \mathrm{PT}$ results can indeed be unnaturally large, especially in the forward kinematics. We argue that higher-order effects of the $\Delta(1232)$ excitation can more naturally be accommodated in the $\mathrm{B} \chi \mathrm{PT}$ calculation. This is due to partial cancellation of the relativistic and $\Delta$-excitation effects which is explicit in the covariant calculation. In contrast to the $\mathrm{HB} \chi \mathrm{PT}$ approach, in $\mathrm{B} \chi \mathrm{PT}$ the effect of $\Delta(1232)$ appears to be compatible both with the Compton scattering and pion photoproduction data.

\section{Acknowledgments}

This work is partially supported by the European Community Research Infrastructure Activity under the FP6 "Structuring the European Research Area" programme (HadronPhysics, contract RII3-CT-2004-506078).

\section{References}

[1] F. E. Low, Scattering Of Light Of Very Low Frequency By Systems Of Spin 1/2, Phys. Rev. 96, 1428 (1954); M. Gell-Mann and M. L. Goldberger, Scattering Of Low-Energy Photons By Particles Of Spin 1/2 Phys. Rev. 96, 1433 (1954).

[2] F. J. Federspiel et al., The Proton Compton effect: A Measurement of the electric and magnetic polarizabilities of the proton, Phys. Rev. Lett. 67, 1511 (1991).

[3] A. Zieger, R. Van de Vyver, D. Christmann, A. De Graeve, C. Van den Abeele and B. Ziegler, 180-degrees Compton scattering by the proton below the pion threshold, Phys. Lett. B 278, 34 (1992).

[4] E. L. Hallin et al., Compton scattering from the proton, Phys. Rev. C 48, 1497 (1993).

[5] B. E. MacGibbon, G. Garino, M. A. Lucas, A.M. Nathan, G. Feldman and B. Dolbilkin, Measurement of the electric and magnetic polarizabilities of the proton, Phys. Rev. C 52, 2097 (1995).

[arXiv:nucl-ex/9507001].

[6] V. Olmos de Leon et al., Low-Energy Compton Scattering And The Polarizabilities Of The Proton, Eur. Phys. J. A 10, 207 (2001).

[7] D. Drechsel, B. Pasquini and M. Vanderhaeghen, Dispersion relations in real and virtual Compton scattering, Phys. Rept. 378, 99 (2003). [arXiv: hep-ph/0212124].

[8] M. Schumacher, Polarizability of the nucleon and Compton scattering, Prog. Part. Nucl. Phys. 55, 567 (2005). [arXiv: hep-ph/ 0501167 ].

[9] S. Weinberg, Phenomenological Lagrangians, Physica A 96, 327 (1979).

[10] J. Gasser and H. Leutwyler, Chiral Perturbation Theory To One Loop, Annals Phys. 158, 142 (1984);

[11] V. Bernard, N. Kaiser and U.-G. Meißner, Chiral expansion of the nucleon's electromagnetic polarizabilities, Phys. Rev. Lett. 67, 1515 (1991); Nucleons with chiral loops: Electromagnetic polarizabilities, Nucl. Phys. B 373, 346 (1992).

[12] V. Bernard, N. Kaiser, and U.-G. Meißner, Chiral dynamics in nucleons and nuclei, Int. J. Mod. Phys. E 4, 193 (1995). [arXiv: hep-ph/9501384]. 
[13] T. R. Hemmert, B. R. Holstein and J. Kambor, Delta(1232) and the Polarizabilities of the Nucleon, Phys. Rev. D 55, 5598 (1997). [arXiv: hep-ph/9612374].

[14] W. M. Yao et al. [Particle Data Group], Review of particle physics, J. Phys. G 33, 1 (2006).

[15] E. Jenkins and A. V. Manohar, Baryon Chiral Perturbation Theory Using A Heavy Fermion Lagrangian, Phys. Lett. B 255, 558 (1991).

[16] J. Gasser, M. E. Sainio and A. Svarc, Nucleons With Chiral Loops, Nucl. Phys. B 307, 779 (1988).

[17] J. A. McGovern, Compton scattering from the proton at fourth order in the chiral expansion, Phys. Rev. C 63, 064608 (2001) [Erratum-ibid. 66, 039902 (2002)]. [arXiv: nucl-th/0101057].

[18] S. R. Beane, M. Malheiro, J. A. McGovern, D. R. Phillips and U. van Kolck, Nucleon polarizabilities from low-energy Compton scattering, Phys. Lett. B 567, 200 (2003) [Erratum-ibid. 607, 320 (2005)]; [arXiv:nucl-th/0209002]. Compton scattering on the proton, neutron, and deuteron in chiral perturbation theory to $O\left(Q^{4}\right)$, Nucl. Phys. A 747, 311 (2005). [arXiv: nucl-th/ 0403088 ].

[19] J. Gegelia and G. Japaridze, Matching heavy particle approach to relativistic theory, Phys. Rev. D 60, 114038 (1999); J. Gegelia, G. Japaridze and X. Q. Wang, J. Phys. G 29, 2303 (2003).

[20] T. Fuchs, J. Gegelia, G. Japaridze and S. Scherer, Renormalization of relativistic baryon chiral perturbation theory and power counting, Phys. Rev. D 68, 056005 (2003).

[21] T. Becher and H. Leutwyler, Baryon chiral perturbation theory in manifestly Lorentz invariant form, Eur. Phys. J. C 9, 643 (1999). [arXiv: hep-ph/9901384].

[22] A. I. L'vov, A Dispersion look at the chiral perturbation theory: Nucleon electromagnetic polarizabilities, Phys. Lett. B 304, 29 (1993).

[23] V. Pascalutsa, B. R. Holstein and M. Vanderhaeghen, A derivative of the Gerasimov-Drell-Hearn sum rule, Phys. Lett. B 600, 239 (2004). [arXiv: hep-ph/ 0407313 ].

[24] B. R. Holstein, V. Pascalutsa and M. Vanderhaeghen, Sum rules for magnetic moments and polarizabilities in QED and chiral effective-field theory, Phys. Rev. D 72, 094014 (2005).

[25] R. P. Hildebrandt, H. W. Griesshammer, T. R. Hemmert and B. Pasquini, Signatures of chiral dynamics in low energy Compton scattering off the nucleon, Eur. Phys. J. A 20, 293 (2004).

[26] V. Pascalutsa and D. R. Phillips, Effective theory of the Delta(1232) in Compton scattering off the nucleon, Phys. Rev. C 67, 055202 (2003). [arXiv: nucl-th/ 0212024 ].

[27] V. Lensky and V. Pascalutsa, Manifestly-covariant chiral PT calculation of nucleon Compton scattering, JETP Lett. 89 (2009) 108 [arXiv: 0803.4115 [nucl-th] ].

[28] A. Metz and D. Drechsel, Generalized polarizabilities of the nucleon studied in the linear sigma model, Z. Phys. A 356, 351 (1996).

[29] A. I. L'vov, Compton Scattering On Proton At Energies Up To 400-Mev And Finite Energy Sum Rules, Sov. J. Nucl. Phys. 34, 597 (1981)

[30] A. I. L'vov, V. A. Petrun'kin and M. Schumacher, Dispersion Theory Of Proton Compton Scattering In The First And Second Resonance Regions, Phys. Rev. C 55, 359 (1997).

[31] V. Pascalutsa and M. Vanderhaeghen, Electromagnetic nucleon to Delta transition in chiral effective field theory, Phys. Rev. Lett. 95, 232001 (2005); Chiral effective-field theory in the Delta(1232) region. I: Pion electroproduction on the nucleon, Phys. Rev. D 73, 034003 (2006).

[32] V. Pascalutsa, M. Vanderhaeghen and S. N. Yang, Electromagnetic excitation of the Delta(1232) resonance, Phys. Rept. 437, 125 (2007). [arXiv: hep-ph/0609004]. 